\section{EDITORIAL}

TOMO 59

Número 8

Octubre

2006
ARCHIVOS ESPAÑOLES DE UROLOGIA

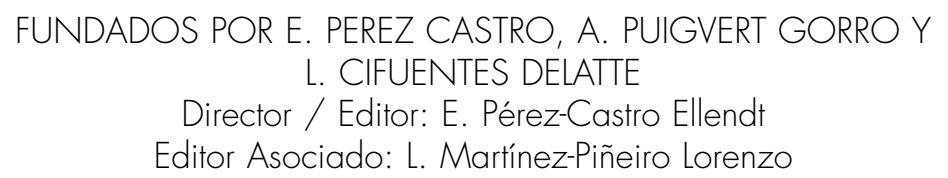

FUNDADOS POR E. PEREZ CASTRO, A. PUIGVERT GORRO Y

Director / Editor: E. Pérez-Castro Ellendt

Editor Asociado: L. Martínez-Piñeiro Lorenzo

\title{
La indicación terapéutica: ¿es una categoría inmutable?
}

\section{Antoni Gelabert Mas}

Servicio y Cátedra de Urología y Unidad Quirúrgica de Trasplante Renal. Hospital del Mar. Universitat Autònoma de Barcelona. Barcelona. España.

Los tratados clásicos de licenciatura de - El autor es Catedrático de Urología de la Universidad Autónoma de Barcelona y Jefe del Servicio de Urología del Hospital del Mar, de Barcelona. Ha sido Director-Coordinador de la Unidad Docente de la Facultad de Medicina en el IMAS. Se reconoce en sus maestros de formación: Prof. J.M. ${ }^{\mathrm{a}}$ Gil Vernet (Barcelona), el Prof. R. Küss (París), el Prof. P. Carretero (Barcelona) y el Prof. W. Gregoir (Bruselas), de quienes aprendió el detalle de la técnica quirúrgica, el rigor clínico y la insatisfacción científico-intelectual permanente.

- La actividad asistencial de su Servicio de Urología está sometida a una gran presión y descansa sobre un grupo de colaboradores altamente cualificados, lo que permite mantener en formación un número de MIR ascendente y asu- 
mir responsablemente los becarios extranjeros aceptados.

- Ha publicado más de 250 artículos en revistas indexadas nacionales y extranjeras; $y$ varias decenas de artículos de divulgación y editoriales. Es autor de 15 capítulos de libros impresos. Editor/Director de una revista científica sobre UroOncología y también editor de varios tratados sobre Urología.

- Su dedicación personal preferente es la Uro-Oncología, liderando un grupo multidisciplinario e interhospitalario muy activo y codirigiendo la Unidad Funcional de Urología Oncológica del Hospital del Mar. Actualmente es el Coordinador Nacional del Grupo de Urología Oncológica de la A.E.U.

- Ha dirigido 20 tesis y se están realizando varias actualmente bajo su dirección. La investigación básica se realiza en un grupo multidisciplinario centralizado en el I.B.F. (Instituto de Biología FundamentalUAB) con becarios en formación predoctoral y dedicada específicamente a la Citogenética y Biología Molecular de los tumores urológicos. Ha creado junto a patólogos y biólopos el LUPA (Laboratori de Uro-Patología Aplicada) en el Hospital del Mar en el que se estan desarrollando varias líneas de investigación sobre la misma temática. ha hecho aparecer los conceptos de cirugía mínimamente invasiva (CMI) y cirugía mayor ambulatoria (CMA), y ha obligado a definir áreas especiales en los hospitales para estos pacientes, los que son controlados un tiempo limitado posteriormente al procedimiento quirúrgico y a las pocas horas remitidos a su domicilio. Tales procedimientos reportan a parte de un menor coste sanitario, una mejor calidad de vida en el postoperatorio a los pacientes a los que se les aplican estas técnicas, pero además se benefician de ellas unos grupos especiales de pacientes que por su pluripatología no eran candidatos a ciertas técnicas terapéuticas cuya agresividad biológica y co-morbilidad habitual obligaba hasta ayer mismo a estar proscritas en estos grupos de pacientes, ya que les hubiere llevado, a una situación vital límite cuando no al éxitus.

Un ejemplo demostrativo: quistes renales clasificados como Bosniak 2F, asintomáticos y diagnosticados de manera casual; tan solo unos pocos años atrás la ortodoxia asistencial indicaba una abstención terapéutica y proponía seguimiento clínico y por imagen. En caso de intervenir, se hubiera practicado una lumbotomía exploradora lo que suponía añadir una considerable morbilidad. Con estas razones podemos decir que el binomio coste/beneficio no estaba balanceado. Pero, ¿y si la acción terapéutica hubiera consistido, como sería actualmente, en una retroperitoneoscopia laparoscópica?, nuestra indicación terapéutica ¿sería idéntica desde el primer momento a seguimiento clínico y por imagen?. En mi opinión, claramente no. Hoy ante un diagnóstico de quiste renal Bosniak 2F, nos inclinaríamos muy probablemente por la opciónlaparoscópica de entrada, la que puede ser efectuada como CMI, y para el paciente supone una mínima morbilidad.

Otro supuesto diagnóstico: HBP grado IV en pacientes con patología asociada cardiorrespiratoria que supongan un ASA IV; en tales 
situaciones clínicas la práctica de una RTU, aún siendo una procedimiento que podriamos etiquetar de minimamente invasivo, existe riesgo de absorción de líquido de irrigación con la correspondiente alteración hidroelectrolítica, duración excesivamente larga del proceso, bajada significativa de hematocrito, etc; desde el servicio de Anestesiología muy probablemente se hubiera tomado la decisión de abstención terapèutica, por el riesgo/cierto de complicaciones severas, incluido el éxitus.

Actualmente en tales supuestos clínicos puede efectuarse la intervención mediante Láser. Con este procedimiento no hay alteración hidroelectrolítica aún en el caso de absorción de líquido de lavado, muy poco probable, ya que se utiliza suero fisiológico, y por ello la absorción es fácilmente manejable mediante la administración de diuréticos; no existe disminución significativa de hematocrito por lo que la descompensación cardíaca es prácticamente imposible por esta causa; la duración del procedimiento, aún siendo casi idéntica con la nucleación endoscópica (HoLep) - bastante mayor en caso de adenomas de más de $100 \mathrm{gr}$ y ser intervenidos por vaporización, que con una RTU clásica. Al existir una monitorización por parte del servicio de Anestesiología sin alteraciones significativas, no supone impedimento terapéutico, es decir, se amplia la indicación a pacientes que antes hueran sido rechazados precisamente por su indicación terapéutica.

Somos conscientes que a pesar de todo tales procedimientos pueden generar graves consecuencias cuando existe un fallo de los mismos, pero deben aplicarse para ir avanzado en medicina.

Y así de esta manera podríamos ir desglosando patologías en las que el advenimiento de técnicas menos invasivas, así como la mejora de los procedimientos anestésicos, ha hecho cambiar la indicación terapéutica. Es decir, estamos frente a una modificación conceptual de una categoría, en este caso indicación/abstención quirúrgica, que pasa de ser inmutable a ser discutible.

Siempre habrá situaciones que nos coloquen al fino de lo imposible, pero nuestra responsabilidad científica y social exige asumirlas ya que es obligado para avanzar. 